\title{
Non-Hypoellipticity for Degenerate Elliptic Operators
}

\author{
Dedicated to the memory of Professor C. Goulaouic
}

By

Yoshinori MORIMOTO*

\section{Introduction}

Let $L_{\infty}$ be a partial differential operator in $R^{3}$ of the form

$$
L_{\infty}=D_{x}^{2}+\phi(x)^{2} D_{y}^{2}+D_{t}^{2}, \quad D_{x}=\frac{1}{i} \frac{\partial}{\partial x}, \cdots, \cdots,
$$

where $\phi \in C^{\infty}, \phi(0)=0, \phi(x)>0(x \neq 0), \phi(x)=\phi(-x)$ and $\phi$ is non-decreasing in $[0, \infty)$. In the recent paper [6], Kusuoka-Strook have shown that $L_{\infty}$ is $C^{\infty}$-hypoelliptic in $R^{3}$ if and only if $\phi(x)$ satisfies

$$
\lim _{x \downarrow 0} x \log \phi(x)=0 .
$$

If $\phi(x)=\exp \left(-1 /|x|^{\sigma}\right)$ for $\sigma>0$ the condition (1) means $\sigma<1$. This result was given as an application of the Malliavin calculus (, which is a theory about stochastic differential equations). The purpose of the present paper is to show the necessity of the condition (1) for $C^{\infty}$-hypoellipticity of $L_{\infty}$ by another simple method.

The method used here is analogous to the one of Bouendi-Goulaouic [1], where nonanalytic hypoellipticity of the operator $L_{1}=D_{x}^{2}+x^{2} D_{y}^{2}+D_{t}^{2}$ was proved. In [1], a solution $u$ of $L_{1} u=0$ was constructed in the form

$$
u(x, y, t)=\sum_{N=0}^{\infty} t^{2 N} A\left(x, D_{x}, D_{y}\right)^{N} w(x, y) /(2 N) !,
$$

where $A=L_{1}-D_{t}^{2}$ and $w(x, y)(=u(x, y, 0))$ is nonanalytic $C^{\infty}$-function defined in a neighborhood $W$ of the origin in $R_{x, y}^{2}$ and satisfies for any integer $N>0$

$$
\left\|A\left(x, D_{x}, D_{y}\right)^{N} w(x, y)\right\|_{L^{2}(W)} \leqq C^{N+1}(2 N) ! 。
$$

Here $C$ is a positive constant independent of $N$. The estimate (3) implies that $u$ is well-defined as an $L^{2}(W)$-valued analytic function with respect to $t \in(-\delta, \delta)$

Communicated by S. Matsuura, June 5, 1985.

* Department of Engineering Mathematics, Faculty of Engineering, Nagoya University, Nagoya 464, Japan. 
for a small $\delta>0$.

In the present paper, assuming that the condition (1) is not fulfilled we construct a solution $u \notin C^{\infty}$ of $L_{\infty} u=0$ which has the same form as (2). To find a convenient function $w(x, y) \notin C^{\infty}$ satisfying (3) we consider an eigenvalue problem in an interval $[-1,1]$ with the Dirichlet boundary condition for an ordinary differential operator $A_{\infty}\left(x, D_{x}, \eta\right)=-\frac{d^{2}}{d x^{2}}+\phi(x)^{2} \eta^{2}$ with a parameter $\eta \neq 0$. This point of view permits to extend the result of [1]. Namely, we can also show nonanalytic hypoellipticity of operators $L_{k}=D_{x}^{2}+x^{2 k} D_{y}^{2}+D_{t}^{2}, k=2,3, \cdots$ (cf. [9]). We remark that the method of the present paper is applicable to show non-hypoellipticity of degenerate elliptic operators of higher order than 2 , differing from that of [6].

As to the operator $L_{\infty}$, it should be noted that an operator $A_{\infty}=D_{x}^{2}+\phi(x)^{2} D_{y}^{2}$ is $C^{\infty}$-hypoelliptic in $R_{x, y}^{2}$ without the condition (1) (Fedii [3], cf. [10]). We remark that $L_{\infty}$ and $A_{\infty}$ with infinite degeneracy do not satisfy Hörmander's sufficient condition for $C^{\infty}$-hypoellipticity in $R^{3}$ and $R^{2}$, respectively ([4]).

The author would like to thank gratefully Prof. T. Matsuzawa for useful discussions. The author also wishes to express his hearty gratitude to Prof. $\mathrm{H}$. Tamura for his helpful suggestion to the eigenvalue problem.

\section{$\S 1$. Main Results}

Let $L$ be a differential operator in $R^{3}$ of the form

$$
L=D_{x}^{2}+g(x) D_{y}^{2}+D_{t}^{2},
$$

where $g(x) \in C^{\infty}$ satisfies $g(x) \geqq 0$ and $g(0)=0$.

Theorem 1. Assume that $g(x)$ satisfies

$$
\liminf _{x \rightarrow 0}|x \log g(x)| \neq 0,
$$

or

$$
\left\{\begin{array}{l}
\limsup _{x \downarrow 0}|x \log g(x)| \neq 0, \\
g(x)=g(-x) \text { and } g(x) \text { is non-decreasing in }[0, \infty) .
\end{array}\right.
$$

Then $L$ is not $C^{\infty}$-hypoelliptic in $R^{3}$. More precisely, one can find a function $u$ defined in some neighborhood $V$ of the origin, belonging to $L^{2}(V)$, not to $C^{\infty}$ and such that $L u=0$.

Remark 1. The condition (1) is equivalent to $\limsup _{x \downarrow}|x \log \phi(x)|=0$. The operator $L$ of (4) is more general than $L_{\infty}$ because $g$ is not always expressed in the form $g=\phi^{2}$ for a non-negative $C^{\infty}$-function $\phi$ (see Remark 2 of Theorem 1.1 of [10]). 
Remark 2. The solution $u$ will be constructed as an $L^{2}(W)$-valued analytic function with respect to $t \in(-\delta, \delta)$, where $W=(-1,1) \times R_{y}^{1}$ and $\delta$ is a small positive constant.

Let $\gamma^{(s)}(\Omega)$ for real $s \geqq 1$ denote a class of Gevrey function of order $s$ defined in an open set $\Omega$. (Here $\gamma^{(1)}(\Omega)$ denotes a class of analytic functions in $\Omega$ ). We say that a differential operator $L$ is $\gamma^{(s)}$-hypoelliptic in $R^{3}$ if and only if for any open set $\Omega$ of $R^{3}$ we have

$$
u \in \mathscr{D}^{\prime}(\Omega), \quad L u \in \gamma^{(s)}(\Omega) \Rightarrow u \in \gamma^{(s)}(\Omega) .
$$

Theorem 2. Assume that $g(x)$ equals $x^{2 k}, k=1,2, \cdots$, that is, the operator $L=L_{k}(k=1,2, \cdots)$. Then $L$ is not $\gamma^{(s)}$-hypoelliptic in $R^{3}$ for any s such that $1 \leqq s<k+1$ (, and hence $L$ is not analytic hypoelliptic). More precisely, for any $1 \leqq s<k+1$ one can find a function $u$ defined in some neighborhood $V$ of the origin, belonging to $\gamma^{(k+1)}(V)$, not to $\gamma^{(s)}(V)$ and such that $L u=0$.

Remark 3. It is well-known that $A_{k}=D_{x}^{2}+x^{2 k} D_{y}^{2}$ is analytic hypoelliptic in $R^{2}$ for any $k=1,2, \cdots$ ([7]). Recently, Matsuzawa [8] has shown that $L_{k}$ is $\gamma^{(k+1)}$-hypoelliptic in $R^{3}$, more precisely, $L_{k}$ is partially $\gamma^{(k+1)}$-hypoelliptic with respect to $y$ variable and partially analytic hypoelliptic with respect to $x$ and $t$ variables (cf. Derridj-Zuily [2]).

Remark 4. Métivier [9] independently proved non $\gamma^{(k+1)}$-hypoellipticity of $L_{k}$ in more general form (see Theorem 3.5 and Corollary 3.7 of [9]). In [9], the existence of $w(x, y)$ satisfying (3) is reduced to the subelliptic estimate instead of the eigenvalue problem.

Theorem 3. Let $l, m$ and $n$ be positive integers and let $\tilde{L}$ be a differential operator of the form

$$
\widetilde{L}=D_{x}^{2 l}+g(x) D_{y}^{2 m}+D_{t}^{2 n},
$$

where $g(x) \in C^{\infty}$ satisfies $g \geqq 0$ and $g(0)=0$. If $g(x)$ satisfies

$$
\liminf _{x \rightarrow 0}\left|x^{l / n} \log g(x)\right| \neq 0
$$

or

$$
\left\{\begin{array}{l}
\limsup _{x \downarrow 0}\left|x^{l / n} \log g(x)\right| \neq 0, \\
g(x)=g(-x) \text { and } g(x) \text { is non-decreasing in }[0, \infty),
\end{array}\right.
$$

then $\tilde{L}$ is not $C^{\infty}$-hypoelliptic in $R^{3}$.

Remark 5. If $g(x)$ equals $\exp \left(-1 /|x|^{\sigma}\right)$ then the condition (6)' means $\sigma \geqq l / n$. We remark that an operator $D_{x}^{2 l}+\exp \left(-1 /|x|^{\sigma}\right) D_{y}^{2 m}$ is $C^{\infty}$-hypoelliptic in $R^{2}$ for any $\sigma>0$ (see $[10]$ ). 


\section{§2. Proofs}

We begin with the proof of Theorem 1 in the case that $g(x)$ satisfies the condition (5). Note that $\log g(x)$ is negative for $|x|$ small enough. The condition (5) implies that there exists a constant $\delta_{0}>0$ such that

$$
g(x) \leqq \exp \left(-\delta_{0} /|x|\right)
$$

if $x$ belongs to a small neighborhood of the origin. Consider an eigenvalue problem in an interval $I_{a}=(-a, a) \subset R_{x}^{1}(a>0)$

$$
\left\{\begin{array}{l}
A\left(x, D_{x}, \eta\right) v(x, \eta) \equiv\left(-d^{2} / d x^{2}+g(x) \eta^{2}\right) v(x, \eta)=\lambda v(x, \eta), \\
v(a, \eta)=v(-a, \eta)=0
\end{array}\right.
$$

where $\eta$ is the dual variable of $y$ and considered as a parameter for a while. Since $A$ is a selfadjoint operator that is bounded from below, it follows from Theorem XIII. 1 of [11] that the minimal eigenvalue $\lambda_{0}(a, \eta)$ is given by the formula

$$
\lambda_{0}(a, \eta)=\inf _{\substack{f \in C_{0}^{\infty}(I a) \\\|f\|_{L^{2}}=1}}(A f, f)_{L^{2}}>0 .
$$

In view of (9), it is clear that $\lambda_{0}(a, \eta) \leqq \lambda_{0}\left(a_{0}, \eta\right)$ if $a \geqq a_{0}$. Set $a_{0}=\delta_{0} / 2 \log |\eta|$ and assume $|\eta|$ large enough. Then it follows from (7) that $g(x) \eta^{2} \leqq 1$ for $x \in I_{a_{0}}$. Let $\tilde{\lambda}_{0}(a)$ denote the minimal eigenvalue of the eigen value problem (8) with $A$ replaced by $-d^{2} / d x^{2}+1$. Comparing (9) and a similar formula for $\tilde{\lambda}_{0}\left(a_{0}\right)$ we have $\lambda_{0}\left(a_{0}, \eta\right) \leqq \tilde{\lambda}_{0}\left(a_{0}\right)$ for large $|\eta|$. Since $\tilde{\lambda}_{0}\left(a_{0}\right)$ equals $C^{\prime} a_{0}^{-2}$ for a constant $C^{\prime}$ independent of $\eta$ we have

$$
0<\lambda_{0}(1, \eta) \leqq C^{\prime \prime}(\log |\eta|)^{2} \quad \text { for large }|\eta|,
$$

where $C^{\prime \prime}$ is a constant independent of $\eta$. Let $v_{0}(x, \eta)$ be an eigenfunction associated with $\lambda_{0}(1, \eta)$ such that $\left\|v_{0}(x, \eta)\right\|_{L^{2}\left(I_{1}\right)}=1$. Take a function $\phi(y) \in L^{2}$ satisfying for a constant $c_{0}>0$

$$
c_{0}\langle\eta\rangle^{-2} \leqq|\hat{\psi}(\eta)| \leqq c_{0}^{-1}\langle\eta\rangle^{-2},\langle\eta\rangle=\sqrt{1+|\eta|^{2}},
$$

where $\hat{\phi}(\eta)$ denotes the Fourier transform of $\phi(y)$. Set

$$
w(x, y)=\int \exp (i y \cdot \eta) v_{0}(x, \eta) \hat{\psi}(\eta) d \eta / 2 \pi
$$

Then it is clear that $w(x, y) \oplus C^{\infty}$. Furthermore, we see that $w$ satisfies the estimate (3) with $W=(-1,1) \times R_{y}^{1}$ for any $N=1,2, \cdots$. In fact, it follows from $A\left(x, D_{x}, \eta\right)^{N} v_{0}(x, \eta)=\lambda_{0}(1, \eta)^{N} v_{0}(x, \eta)$ that the estimate 


$$
\begin{aligned}
\left\|A\left(x, D_{x}, D_{y}\right)^{N} w(x, y)\right\|_{L^{2}\left(I_{1} \times R_{y}\right)} & =\left\|\lambda_{0}(1, \eta)^{N} \eta_{0}(x, \eta) \hat{\psi}(\eta)\right\|_{L^{2}\left(I_{1}>R_{r_{i}}\right)} \\
& =\left\|\lambda_{0}(1, \eta)^{N} \hat{\psi}(\eta)\right\|_{L^{2}\left(R_{\eta}\right)} \\
& \leqq C_{1}^{N}\left\|(\log \langle\eta\rangle)^{2 N}\langle\eta\rangle^{-2}\right\|_{L^{2}\left(R_{\eta}\right)} \\
& \leqq C_{2}^{N+1}(2 N) !
\end{aligned}
$$

holds for constants $C_{1}$ and $C_{2}$ independent of $N$. Here in order to get the last inequality, we used an elementary inequality $s^{2 N} e^{-s / 2} \leqq 4^{N}(2 N)$ ! ( $(\geqq 0)$ by setting $s=\log \langle\eta\rangle$. Define a function $u$ by the formula (2) with $A=D_{x}^{2}+g(x) D_{y}^{2}$. Then the estimate (12) shows that $u$ is well-defined as an $L^{2}\left((-1,1) \times R_{y}\right)$-valued analytic function with respect to $t \in(-\delta, \delta)$ for a small $\delta>0$. Since $u(x, y, 0)$ $=w(x, y)$ we see $u \notin C^{\infty}$. This concludes the proof of Theorem 1 when $g(x)$ satisfies the condition (5).

If $g(x)$ satisfies the condition (5)' there exist a $\delta_{1}>0$ and a decreasing sequence of positive numbers $\left\{a_{\jmath}\right\}_{j=1}^{\infty}$ such that $\lim _{j \rightarrow \infty} a_{\jmath}=0$ and $-a_{j} \log g\left(a_{\jmath}\right) \geqq \delta_{1}$. Since $g(x)=g(-x)$ and $g(x)$ is non-decreasing in $[0, \infty)$, it follows that

$$
\begin{aligned}
g(x) \eta^{2} \leqq g\left(a_{j}\right) \eta^{2} \leqq 1 \quad \text { for } \quad x \in\left[-a_{\jmath}, a_{\jmath}\right] \\
\text { if }|\eta| \leqq \exp \left(\delta_{1} / 2 a_{\jmath}\right) .
\end{aligned}
$$

Replacing $a_{0}$ in the above by $a_{\text {, we }}$ see that the minimal eigenvalue $\lambda_{0}(1, \eta)$ satisfies

$$
\lambda_{0}(1, \eta) \leqq \tilde{\lambda}_{0}\left(a_{\jmath}\right)=C^{\prime} a_{\jmath}^{-2} \quad \text { if } \quad|\eta| \leqq \exp \left(\delta_{1} / 2 a_{\jmath}\right),
$$

where $C^{\prime}$ is the absolute constant. Therefore, we get

$$
\lambda_{0}(1, \eta) \leqq \tilde{C}(\log |\eta|)^{2} \quad \text { if } \quad \exp \left(\delta_{1} / 3 a_{\jmath}\right) \leqq|\eta| \leqq \exp \left(\delta_{1} / 2 a_{\jmath}\right),
$$

where $\tilde{C}$ is a constant independent of $|\eta|$ and $j$. Take a function $\phi(y) \in L^{2}$ satisfying.

$$
\left\{\begin{array}{l}
c_{1}\langle\eta\rangle^{-2} \leqq \hat{\psi}(\eta) \leqq c_{1}^{-1}\langle\eta\rangle^{-2} \quad\left(c_{1}>0\right) \\
\text { if } \exp \left(\delta_{1} / 3 a_{\jmath}\right) \leqq|\eta| \leqq \exp \left(\delta_{1} / 2 a_{\jmath}\right), \\
\hat{\phi}(\eta)=0 \quad \text { otherwise. }
\end{array}\right.
$$

Using $(10)^{\prime}$ and $(11)^{\prime}$ in place of (10) and (11), respectively, we have the estimate (12), which completes the proof of Theorem 1 in the case that $g(x)$ satisfies the condition (5)'.

Theorem 2 can be proved by the same way as in the proof of Theorem 1 . If we set $a_{0}=|\eta|^{-1 /(k+1)}$ we have $g(x) \eta^{2}=x^{2 k} \eta^{2} \leqq|\eta|^{2 /(k+1)}$ for $x \in I_{a_{0}}$. Considering a "majorant" eigenvalue problem in $I_{a_{0}}$ for an operator $-(d / d x)^{2}+$ $|\eta|^{2 /(k+1)}$, we also see that the minimal eigenvalue of (8) satisfies

$$
0<\lambda_{0}(1, \eta) \leqq C_{3}|\eta|^{2 /(k+1)} \quad \text { for large }|\eta|,
$$

where $C_{3}$ is a constant independent of $\eta$. For a fixed $s$ satisfying $1 \leqq s<k+1$ take a function $\phi(y) \in \gamma^{(k+1)} \cap C_{0}^{\infty}$ such that $\phi(y) \notin \gamma^{(s)}$. Since $\phi \in \gamma^{(k+1)} \cap C_{0}^{\infty}$ it 
follows that

$$
|\hat{\psi}(\eta)| \leqq \varepsilon^{-1} \exp \left(-\varepsilon\langle\eta\rangle^{1 /(k+1)}\right)
$$

for a constant $\varepsilon>0$ independent of $\eta$. Using (13) and (14) in place of (10) and (11), respectively, we obtain the estimate (3), which shows the existence of the desired solution $u$ of $L_{k} u=0$.

The proof of Theorem 3 is also parallel to that of Theorem 1. For the proof it suffices to consider an eigenvalue problem with the Dirichlet boundary condition for an operator $\tilde{A}\left(x, D_{x}, \eta\right)=\left(-d^{2} / d x^{2}\right)^{l}+g(x) \eta^{2 m}$ instead of (8) and to replace (2) and (3) by

$$
u(x, y, t)=\sum_{N=0}^{\infty} t^{2 n N}\left((-1)^{n-1} \tilde{A}\left(x, D_{x}, D_{y}\right)\right)^{N} w(x, y) /(2 n N) !
$$

and

$$
\left\|\tilde{A}\left(x, D_{x}, D_{y}\right)^{N} w(x, y)\right\|_{L^{2}(W)} \leqq C^{N+1}(2 n N) !,
$$

respectively. The detail of the proof of Theorem 3 is left to the reader.

\section{References}

[1] Bouendi, M.S. and Goulaouic, C., Nonanalytic hypoellipticity for some degenerate elliptic operators, Bull. Amer. Math. Soc., 78 (1972), 483-486.

[2] Derridj, M. and Zuily, C., Sur la regularité Gevrey des opérateurs de Hörmander, J. Math. pures et appl., 52 (1973), 309-336.

[3] Fediř, V.S., On a criterion for hypoellipticity, Math. USSR SB., 14 (1971), 15-45.

[4] Hörmander, L., Hypoelliptic second order differential equations, Acta Math., 119 (1967), 147-171.

[5] Kusuoka, S., Malliavin calculus and its applications, Sûgaku, 36, Iwanami (1984), 97-109, in Japanese.

[6] Kusuoka, S. and Strook, D., Applications of the Malliavin calculus, Part II, $J$. Fac. Sci. Univ. Tokyo Sect. IA, Math., 32 (1985), 1-76.

[7] Matsuzawa, T., Sur les equations $u_{t t}+t^{\alpha} u_{x x}$, Nagoya Math. J., 42 (1971), 43-55.

[8] - Partial regularity and applications, to appear in Nagoya Math. J.

[9] Métivier, G., Proprieté des itérés et ellipticité, Comm. in Partial Differential Equations, 3 (1978), 827-876.

[10] Morimoto, Y., On the hypoellipticity for infinitely degenerate semi-elliptic operators, J. Math. Soc. Japan, 30 (1978), 327-358.

[11] Reed, M. and Simon, B., Methods of modern mathematical physics IV, Analysis of operators, Academic Press, New York, 1978. 